\title{
E ffect of Low-Intensity Leg Exercise on Ventilatory Threshold during Incremental Arm Exercise
}

\author{
Hisayoshi Ogata, Takahiro Yunoki and Tokuo Yano \\ Laboratory of Human Movement Sciences, Faculty of Education, Hokkaido University
}

\section{Introduction}

It is known that lactate, the level of which is increased during exercise, disappears more rapidly during low-intensity exercise than during passive recovery (Belcastro and Bonen, 1975; Stamford et al., 1981). This is because lactate can be used as an energy source during low-intensity exercise. If lactate can be used in such a way, it is supposed that the lactate that is produced during high-intensity exercise would disappear more rapidly during simultaneous low-intensity exercise. Therefore, in this study, we had subjects perform both incremental arm exercise and low-intensity steady-state leg exercise at the same time. This protocol was adopted for the following reason. During incremental exercise, if lactate elimination is affected by simultaneous leg exercise, it is supposed that the lactate threshold (LT), the point at which lactate production exceeds elimination, would also be affected. However, because arm exercise was performed, it was difficult to obtain blood samples from the fingertip. Therefore, we used respiratory gas kinetics to examine the effect of low-intensity leg exercise on ventilatory threshold (VT) during incremental arm exercise.

\section{Methods}

Eight healthy male subjects with a mean age of 20 1.7 years, a mean weight of $64.7-5.4 \mathrm{~kg}$, and a mean height of $174-4.8 \mathrm{~cm}$ participated in this study. Three experiments were performed: 1 ) incremental leg exercise $(L E), 2)$ incremental arm exercise $(A E)$, and 3 ) incremental arm exercise together with low-intensity steady-state leg exercise (combined exercise: CE). During LE, the subject performed leg cycling in an upright position with his arms at his sides using a cycle ergometer (E rgometer 232C: Combi). First, the subjects remained in a resting state on the ergometer for $5 \mathrm{~min}$. Then, after 4 min of zero-load cycling, the work load was increased by $20 \mathrm{~W}$ per minute until exhaustion. During $A E$, the center of the ergometer (Ergometer 232CXL: Combi) shaft was positioned at the level of the subject's shoulder, and the subject performed arm cranking in an upright position. The incremental load exercise was the same as that during $L E$. The protocol for $C E$ was the same as that for $A E$ except that the subjects performed simultaneous steadystate leg exercise. Steady-state leg exercise was performed at $30 \%$ of the peak oxygen uptake $\left(\mathrm{V}_{2}\right.$ peak $)$ recorded during LE until the arm exercise finished. All exercises were performed at $60 \mathrm{rpm}$. The subject performed each exercise once a day.

Respiratory gas exchange data were collected throughout the 5-min rest period, throughout the exercise test and for $10 \mathrm{~min}$ after the exercise by the breath-bybreath method using a computerized system (Aeromonitor AE-208S: Minato). Then, the average oxygen uptake ( $\left.\mathrm{VO}_{2}\right)$ and carbon dioxide output $\left(\mathrm{VCO}_{2}\right)$ for each $20-\mathrm{sec}$ interval was calculated.

We plotted $\dot{\mathrm{V} C O} \mathrm{O}_{2}$ against $\dot{\mathrm{V}}_{2}$, and took the ventilatory threshold (VT) to be the point at which the $\mathrm{VCO}_{2}$ began to increase rapidly ( $\mathrm{V}$-slope method; Beaver et al., 1986).

We used a paired student's t-test to compare the variables of the two groups. A value of $p<0.05$ was regarded as significant. The strength of the relationships between dependent and independent variables were expressed by pearson's correlation coefficient.

\section{Results}

Table 1 summarizes the average values and standard deviations of work rate and $\mathrm{V}_{2}$ at the VT, work rate at exhaustion (peak work rate), peak oxygen uptake $\left(\dot{\mathrm{VO}}_{2 \text { peak }}\right)$, and the slope of the $\dot{\mathrm{V}} \mathrm{CO}_{2}-\mathrm{VO}_{2}$ relationship obtained below and above VT during all exercise modes. Significant differences $(p<0.01)$ were observed between the slope below VT and the slope above VT during all exercise modes. During $\mathrm{CE}, \mathrm{V}_{2}$ at the VT was higher than that during $A E$ because of $\dot{V O}_{2}$ on steady state-leg exercise. Peak work rate during CE tended to be lower than that during $A E$. However, for 6 out of the 8 subjects, the work rate at the VT during CE was higher than that during $A E$. For the two remaining subjects, the values were slightly 
Table 1 The average values and standard deviation of work rate and $\dot{\mathrm{V}} \mathrm{O}_{2}$ at the VT, work rate at exhaustion (Peak work rate), peak oxygen uptake $\left(\mathrm{V}_{2}{ }_{2 \text { peak }}\right.$ ), and the slope of the $\mathrm{VCO}_{2}-\dot{V} \mathrm{O}_{2}$ relationship obtained below and above VT during all exercise modes

\begin{tabular}{lccc}
\hline & \multicolumn{1}{c}{ LE } & AE & CE \\
\hline Work rate at the VT (watts) & $113-30$ & $68-11$ & $77-17$ \\
$\mathrm{~V}_{2}$ at the VT (I/min) & $1.24-0.29$ & $1.02-0.17$ & $1.79-0.29$ \\
Peak work rate (watts) & $286-16$ & $158-22$ & $148-17$ \\
$\mathrm{VO}_{2 \text { peak }}(\mathrm{I} / \mathrm{min})$ & $2.81-0.31$ & $2.24-0.50$ & $2.62-0.41$ \\
slope $<$ VT & $0.84-0.12$ & $0.87-0.09$ & $0.96-0.10$ \\
slope $>$ VT & $1.30-0.09 * *$ & $1.28-0.11^{* *}$ & $1.47-0.21^{* *}$ \\
\hline
\end{tabular}

**: significant difference $(p<0.01)$ between the slope below VT and the slope above VT. LE: incremental leg exercise. AE: incremental arm exercise. CE: incremental arm exercise together with low-intensity steady state leg exercise (combined exercise). The work rate for CE represents the work rate of the arm exercise during CE.

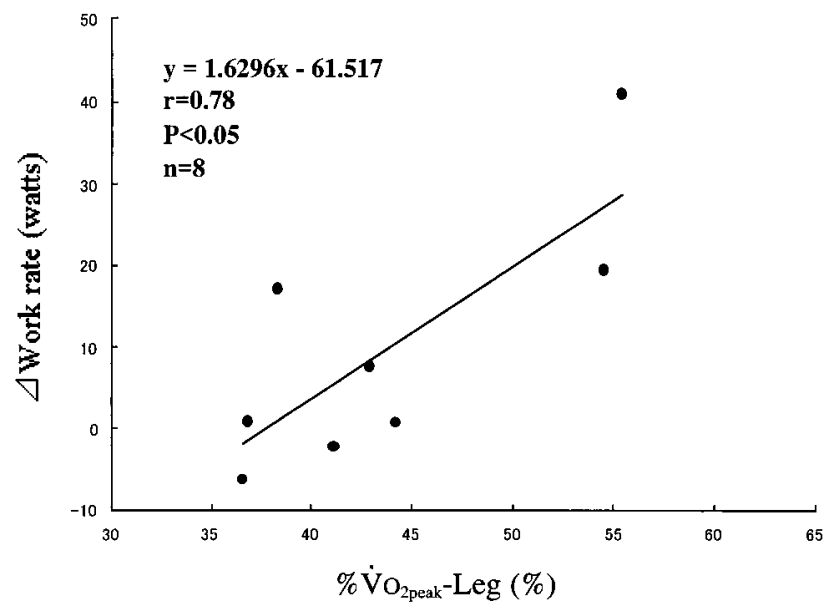

Fig. 1 The difference in work rate at the VT between $A E$ and $\mathrm{CE}$ (Dwork rate) compared with the ratio of $\mathrm{V}_{2}$ at the VT to $\mathrm{V}_{2}$ peak during $\mathrm{LE}\left(\% \mathrm{~V}_{2}\right.$ peak-Leg).

lower during $C E$. Therefore, no significant difference was observed in the work rate at the VT between $A E$ and $C E$. However, when we observed the relationship between the difference in work rate at the VT between $A E$ and $C E$ (Dwork rate) and the ratio of $\mathrm{VO}_{2}$ at the $\mathrm{VT}$ to $\mathrm{VO}_{2}$ peak during $\mathrm{LE}\left(\% \mathrm{VO}_{2}\right.$ peak-Leg), a significant positive correlation $(r=0.78, p<0.05)$ was observed between Dwork rate and $\% \mathrm{VO}_{2 \text { peak }}$-Leg (Fig. 1 ).

\section{Discussion}

Lactate that is generated in active muscles is diffused into the blood, and then taken into liver, inactive muscles or into active muscles performing low-intensity exercise. Throughout this process, lactate is removed from muscle engaged in high-intensity exercise. It is thought that the blood lactate concentration is determined by the rate of systemic lactate production and elimination (Stanley et al., 1985). Since production exceeds elimination, the blood lactate concentration rises during incremental exercise. In this study, we had subjects perform CE under the assumption that if lactate elimination is affected by low-intensity steady-state leg exercise, the VT would rise.

In this study, we set the intensity of the steady-state leg exercise at $30 \% \mathrm{VO}_{2}$ peak recorded during $\mathrm{LE}$. As a result, a rise in the VT was not observed in all subjects. However, it seemed that the higher the VT level was during $L E$, the larger was the rise in the VT during CE. The VT during LE was determined by the rate of systemic lactate production and elimination; the higher the rate of elimination is, the higher the VT can be. Therefore, it is possible that lactate generated during arm exercise can be removed at a higher rate in the subjects who have a higher VT level during LE. On the other hand, it is supposed that since the capacity of the legs for lactate elimination was low, no rise in the VT was observed in subjects whose VT level during $\mathrm{LE}$ was $30-40 \% \mathrm{VO}_{2}$ peak.

It is thought that subjects in whom lactate generation is inhibited also have a higher VT level. Therefore, the rise in the VT level during CE does not necessarily demonstrate that low-intensity leg exercise enhanced lactate elimination. Further study is required to clarify this process.

Acknowledgement. This study was supported by a Grant-in-Aid for Scientific Research (B) from the Ministry of Education, Science, Sports and Culture of Japan (No. 08458013).

\section{References}

Beaver WL, Wasserman K, Whipp BJ (1986) A new method for detecting anaerobic threshold by gas exchange. J Appl Physiol 60: 2020-2027

Belcastro AN, Bonen A ( 1975) Lactic acid removal rates 
during controlled and uncontrolled recovery exercise. J Appl Physiol 39: 932-936

Stamford BA, Weltman A, Moffatt RJ, Sady S (1981) Exercise recovery above and below anaerobic threshold following maximal work. J Appl Physiol 51: 840-844 Stanley WC, Gertz E W, Wisneski J A, Neese RA, Brooks JA (1985) Systemic lactate kinetics during graded exercise in man. Am J Physiol 249: E595-602
Received: April 21, 1999

Accepted: J une 30, 1999

Correspondence to: Hisayoshi Ogata, Kenkoutaiikukagakukouza, Center for Research and Development in Higher E ducation, Hokkaido University, Nishi 8 Kita 17, Kita-ku, Sapporo 060-0817, J apan e-mail: hogata@edu.hokudai.ac.jp 